\title{
Stimuli responsive polymeric nanoparticles in regulated drug delivery for cancer
}

\author{
Deepak Yadav ${ }^{*}$, Suruchi Suri ${ }^{2}$, Anis A Chaudhary ${ }^{3}$, Hemant ${ }^{3}$, Mohd N Beg², Veena Garg ${ }^{1}$, Mohd \\ Asif $^{4}$, Altaf Ahmad ${ }^{3}$
}

${ }^{1}$ Banasthali University, Department of Bioscience and Biotechnology, Rajasthan-304022, India

${ }^{2}$ Department of Pharmaceutics, Faculty of Pharmacy, Jamia Hamdard, Hamdard Nagar, New Delhi 110062, India

${ }^{3}$ Hamdard University, Department of Botany, F/o Science, Hamdard Nagar, New Delhi-110062, India

${ }^{4}$ Hamdard University, Department of Ilmul-Advia, F/o Science, Hamdard Nagar, New Delhi-110062, India

"Corresponding author: e-mail: deepaknano@gmail.com

\begin{abstract}
Stimuli-responsive drug delivery system is a concept in which a drug is delivered at a suitable rate in response to stimuli. States of diseases may cause an alteration in some parameters of the body (e.g. in tumors) and the onset and offset of the drug delivery can be done by using this as a stimuli or a "trigger". Stimuli-responsive ("intellectual" or "sharp") resources and molecules show abrupt property changes in response to miniature changes in external stimuli such as $\mathrm{pH}$, temperature etc. For regulated drug delivery, environmental stimuli such as $\mathrm{pH}$ and temperature, which undertake phase transition in polymer system, have been investigated. Thermally-responsive polymers can be tuned to a preferred temperature variety by copolymerization with a hydrophilic co-monomer or a hydrophobic co-monomer. Hydrophilic co-monomers increase the LCST while hydrophobic co-monomers decrease the LCST. The stimuli responsive polymer for regulated drug delivery can contain a polymer and copolymers having equilibrium of hydrophilic and hydrophobic groups. A number of these polymers have been investigated extensively and some success in drug delivery with them has been achieved, such as polymers and copolymers of $\mathrm{N}$-isopropylacrylamide, PLGA, and PLA, HEMA etc. Thus this review is designed for stimuli $\mathrm{pH}$ and temperature responsive polymeric nanoparticles, which would be helpful to treat various cronic diseases such as cancer and others, for scientists in the field of the regulated drug delivery system.
\end{abstract}

Keywords: Regulated drug delivery, temperature and $\mathrm{pH}$ sensitive nanoparticles, polymeric Micelles, cancer

\section{INTRODUCTION}

Nanotechnology and nanoengineering now have been proved a revolutionary quest to develop and modify almost everything we do, with atomic precision. Nanotechnology, being an interdisciplinary stream, is serving potentially the areas of genetics, biotechnology, physics, chemistry etc. Currently scientists have identified the particular use of nanoparticles as catalysts ${ }^{1}$, photocatalysts, adsorbents, sensors ${ }^{2}$, and ferrofluids. They can be used in optical, electronic, and magnetic devices due to the properties of their materials, and formulation of a different type of stimuli responsive polymers for controlled drug delivery in various diseases, such as cancer, diabetes, filarial, viral infections, bacterial infections etc. WHO has assumed that cancer will be single, important cause with an estimation of 12 million deaths worldwide in 2030. To overcome this disease several therapies like surgery, radiotherapy, immunotherapy, chemotherapy are given to the patients. However, chemotherapy for cancer is usually limited by the toxicity of drugs to normal tissues. Short circulation half-life in plasma, limited aqueous solubility, and non-selectivity are usually encountered by most of the currently available anticancer drugs and thus restrict their therapeutic efficacy ${ }^{3}$. To reduce the toxicity and increase the therapeutic efficacy of anticancer drugs, various drug carriers, such as soluble polymers, polymeric nanoparticles, liposomes, and microspheres have been investigated ${ }^{4,5}$. Many other types of polymeric core-shell nanoparticles have recently appeared to target the hydrophobic, poorly water-soluble and amphiphilic drugs as well as genes, protiens, and enzymes to solid tumour ${ }^{6,7}$.
Human beings possess homeostatic mechanisms to adjust a variety of physiological parameters within an assured range. The parameters include temperature, $\mathrm{pH}$ and salt content. In the event of a disease, however, these parameters become affected due to various mechanisms. It is fundamentally necessary that the body maintains homeostasis for appropriate conditions for enzyme reactions and metabolism to be carried out ${ }^{8,9}$.

In order to deliver such small molecular drugs peptides, proteins, enzymes, hormones and some other hydrophobic drugs at their target specific site, a number of formulations and sources of administration have been developed ${ }^{10}$. Different types of the parental routes of administration contain intravascular (intravenous, intraarterial), intraparenchymal, intramuscular, intradermal, subcutaneous, subdermal, intraperitoneal, intratumor, and intralymphatic injections that hold the use of the syringes, have been used from a long time. The circulatory system provides systemic route to the drugs. Polyethylene glycol (PEG) of different molecular weights and other hydrophilic polymers have given protection to the drugs in the blood environment by avoiding its interaction with blood components and to enhance the prolonged circulation time of the drug by avoiding opsonization, phagocytosis and uptake by the reticuloendothelial system $^{11}$. The regulated release of drugs after their administration is under intensive development. Due to a variety of biologically-liable polymers the drugs have also been complexed to delay their release from depots.

Biodegradable Polymeric nanoparticles of stimuli responsive for regulated drug delivery have evoked great interest in the recent years for clinical administration of drugs ${ }^{12}$. The polymeric nanoparticle systems have 
been found to be useful for stimuli-responsive release of vaccine, for the treatment of inflammation, infection, diabetes, arthritis, impotency, and cancer ${ }^{\mathbf{1 3 , 1 4}}$. The most exciting opportunity in regulated drug delivery systems come through the way of stimuli responsive drug delivery systems which may help to deliver a drug via implantable devices. In recent years scientists have shown keen interest in the issue of delivering the drug precisely to a specific site. The benefit of such formulations contain the sustained drug release action on the lesion, facilitate extravasations into the tumor, reduce side effects of the drugs in the delivery system and high potential to cross the range of physiological barriers ${ }^{\mathbf{1 5}, \mathbf{1 6}}$. However, the advantages may be negotiated by the particles, short dwelling time in the blood system due to the recognition and detain by the macrophages in the mononuclear phagocyte system ${ }^{\mathbf{1 7}}$. It is generally accepted that the phagocytosis outcome from the nanoparticles interface with some blood proteins (opsonization). Therefore, the well-organized prevention of the phagocytosis is to keep away from or reduce the adsorption of the particles onto the phagocytes. Particles indistinguishable to the body defense system, which can avoid being cleared from the blood system, are called theft or extensive circulating particles, which can be realized by attaining tremendously small particle size as well as applying proper matrix material and surface engineering ${ }^{18,19}$. Polymers are macromolecules that exhibit an impressive physiochemical changes in response to small changes in their environment stimuli response. It is proved that the rate of the dissolution of drug particulates can be enhanced by decreasing the particle size. Therefore, methods of making fine and small nanoparticles have been formed, and more efforts have gone into the field of regulated drug delivery by controlling their size and making the stimuli responsive to pharmaceutical compositions. For example, dry milling techniques have been invented to minimize particle size and thereby influence to drug absorption, however, the wet grinding technique is more beneficial for decreasing the particle size ${ }^{20}$. Injectable formulations, based on smart polymers are straightforward to prepare and form implants besides the injection upon administration. Smart polymers are of different types based on external stimulus which they respond to (i.e. temperature, $\mathrm{pH}$, solvent, magnetic field, ions, and pressure $)^{\mathbf{2 1}}$. The development of such type of stimuli responsive polymeric nanoparticles for regulated drug delivery systems has gained attention over the past few years. This interest has been glimmered by the reward which comprises effortlessness of applications. These delivery systems acquire, prolonged drug delivery periods, localized drug delivery for a site-specific achievement, reduce body drug dosage with concurrent diminution in possible objectionable effects which are common to most forms of systemic drug delivery, the non toxic degradability, and better patients' compliance and relief ${ }^{22}$. There are many natural or synthetic polymers which are being used in stimuli responsive drug delivery systems ${ }^{23,24}$. Stimuli responsive co-polymers such as PNIPAm/MAA nanoparticles act as responsive nano valves which give appearance like channels in response to changes in external stimuli like temperature and $\mathrm{pH}$ [Fig 1.].

\section{POLYMERS IN NANOPARTICLES FORMULATION}

A common class of polymers used as a stimuli responsive polymeric nanoparticle system holds at least one moiety including acrylamide, acrylate, acrylic $\operatorname{acid}^{\mathbf{2 5 , 2 6 , 2 7 , 2 8}}$ and at least another one moiety including cellulose and its derivatives ${ }^{29}$, e.g. propylcellulose, ethylcellulose, methoxypropylcellulose, methylcellulose, hydroxypropylmethylcellulose, cellulose nitrate, hydroxylethyl cellulose (HEC) ${ }^{\mathbf{3 0}}$ poly(vinyl alcohol), poly(vinyl chloride), polystyrene, polypropylene, polyethylene-oxides ${ }^{31}$, poly(ethylene-co-vinyl acetate), polyesters, e.g., poly(hydroxyvalerianic acid-co-hydroxy-
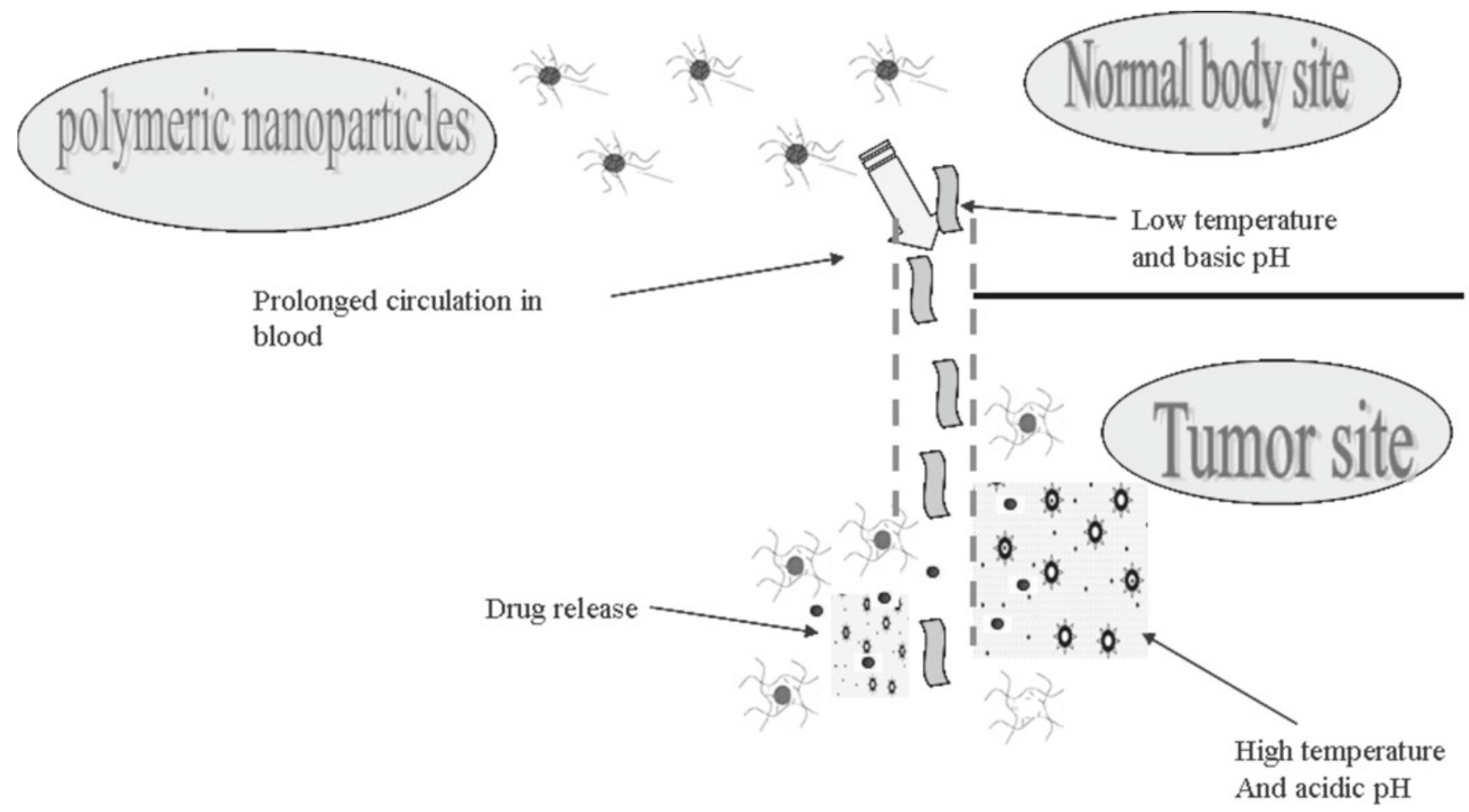

Figure 1. Showing the burst of the stimuli responsive polymeric nanoparticles as regulated drug delivery carriers 
butyric acid), poly(hydroxybutyric acid), poly(lactic acid), poly(lactic acid-co-glycolic acid), poly(glycolic acid), poly(epsilon(-caprolactones), poly(epsilon(-caprolactoneco-DL-lactic acid), polyanhydrides ${ }^{32}$, e.g., poly(maleic anhydride), polyamides, albumin, gelatin, chitosan, collagen, poly (ethylene oxide)-co- $\beta$-benzyl-L-asparate, poly(hydroxyalkyl)-L-glutamines, poly(gamma(-ethylL-glutaminate-co-glutamic acid) ${ }^{33}$, poly(L-leucine-coL-aspartic acid), poly(proline-co-glutamic acid), poly (lactide-co-glycolide), poly(orthoesters), e.g., poly(alkyl 2-cyanoacrylates), polylysin, alginate, alginic acid, polyurethanes, poly(methyl methacrylate), poly(methyl methacrylate-co-methacrylic acid) ${ }^{34}$, poly(methacrylateco-hydroxypropyl-methacrylate $)^{6,35}$ [Fig.2].

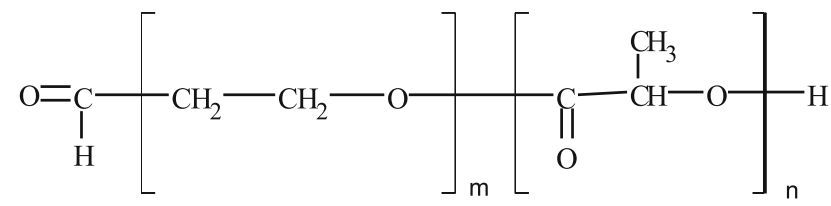

Ploy(ehtylene oxide)-co- $\beta$ - benzyl-L-aspartate

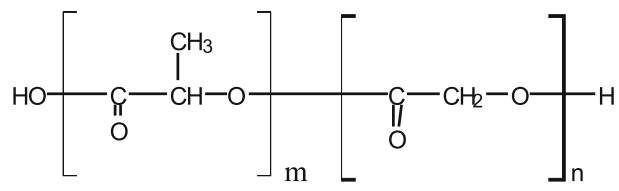

Poly(lactide-co-glycolide)

Figure 2. Structures of poly (ethylene oxide)-co- $\beta$-benzyl-Lasparate and poly (lactide-co-glycolide)

The alterations based on temperature can be utilized to prepare stimulus responsive nanoparticles that are of huge interest in tissue engineering, controlled drug delivery and other areas. The $\mathrm{pH}$-responsive liposomes are colloidal phospholipids vesicles. These vesicles are stable at neutral $\mathrm{pH}$ and rapture under acidic conditions and release the drug in the regions of the body where the $\mathrm{pH}$ is below normal which may be due to several pathological conditions such as inflammation, infection or malignant transformation. However, the tumor interstitium $\mathrm{pH}$ hardly ever turns down below $\mathrm{pH}$ 6.5. Hence in the recent studies it has been proved that the $\mathrm{pH}$ in solid tumors is 0.5 to 1 units lower than in the normal tissue. In the endosomal intracellular compartments $(\mathrm{pH}$ 4.5-6.5) as well as in the tumor and inflammatory tissues $(\mathrm{pH} 6.5)$ it is slightly acidic. This decrease in the $\mathrm{pH}$ stimulates the nanoparticles to burst which causes the release of the drug at specific site of action in the body. This type of stimuli responsive controlled drug delivery system is beneficial for curing the diseases like cancer which affects the homeostatic functions of the body ${ }^{36,37,38}$.

\section{TEMPERATURE RESPONSIVE POLYMERIC NANO- PARTICLES}

A consideration in the building up of temperaturesensitive devices into clinically benefited modulated drug delivery carriers lack in the altering of the temperature of the implanted devices. However, the temperature changes must be very fast to regulate the structural changes in the polymeric nanoparticles and the temperature changes should be localized to the carrier to avoid damage to surrounded normal tissue. Some other methods of changing the temperature are heating pads, non-targeted light and exothermic chemical reactions that have been proposed and are being investigated ${ }^{39,40}$. Stimulus responsive drug delivery permits the release profiles of therapeutic carriers to be configured to match the physiological essentialities of the patients.

Few approaches are introduced to overcome the problems to give the hydrophobic or poorly water-soluble drugs in their encapsulation in polymeric nanoparticles. Polymeric nanoparticles are useful for stimuli regulatory drug delivery as well as for minimizing the adverse effects of the drugs, it maintains the drug efficacy for site specific treatment and increases cell penetration and which ultimately enhances pharmacological activities ${ }^{41,42}$.

Numerous polymers illustrate sharp changes in their solubility as a function of environmental temperature and physical factors ${ }^{43}$. This property is occupied to develop aqueous solutions of such polymers which go through sol-gel transition in response to temperature alteration. Between polymers and water molecules, the interaction forces become unfavorable in contrast to polymerpolymer and water-water interaction at the lower critical solution temperature (LCST) and phase separation takes place as the polymers dehydrates. Consequently, aqueous polymer solutions reveal a spiky enhancement in viscosity, subsequently a tiny temperature increase, forming a semi-solid gel at body temperature. The systems explain high preliminary disintegrated effects which have been credited to the reduction in the volume which gives off a bulky amount of the encapsulated drugs, though. The polymers contain thermosensitive characters are poly (N-isopropylacrylamide) (PNIPAAM), poly (ethylene oxide) - poly (propylene oxide)-poly (ethylene oxide) triblock copolymers (PEO-PPO-PEO), poly (ethylene glycol)-poly (lactic acid)-poly (ethylene glycol) triblocks (PEG-PLAPEG) etc. ${ }^{44}$ There many types of hydrophilic polymeric gels or hydrogels have been investigated. It has been proved that when the polymer temperature is increased to its lower critical solution temperature (LCST), the polymer undergoes reversible phase transition, resulting in the bursting of the polymeric gel configuration $^{45,46,47}$.

Marco Med, a pharmaceutical company introduced triblock copolymer which contains the hydrophobic polyester block and the hydrophilic poly ethylene glycol block. MarcoMed, ReGel ${ }^{\circledR}$, occupies $23 \%(\mathrm{w} / \mathrm{w})$ copolymer of poly (lactide-co-glycolide)-poly (ethylene glycol) - poly (lactide-co-glycolide) (PLGA-PEG-PLGA) in phosphate buffer saline. It is for protein based drugs e.g. insulin. At a concentration of $6 \mathrm{mg} / \mathrm{g} \mathrm{ReGel}{ }^{\circledR}$, composed of paclitaxel, OncoGel ${ }^{\circledR}$, injection releases the drug into the tumor in six weeks ${ }^{\mathbf{4 8 , 4 9}}$.

It has been revealed in recent researches that polymeric nanoparticles (NIPAAM/VP/AA), (NIPAAM/ MMA/AA) LCST $45-50^{\circ} \mathrm{C}$ with a hydrophobic core and a hydrophilic shell are formed from $\mathrm{N}$-isopropyl acrylamide (NIPAAM), at a molar ratio of about $50 \%$ to about $90 \%$; either water-soluble vinyl derivatives like vinylpyrrolidone (VP), or water insoluble vinyl derivatives like methyl methacrylate (MMA), at a molar ratio 
of about $10 \%$ to about $30 \%$; and acrylic acid (AA), at a molar ratio of about $10 \%$ to about $30 \%{ }^{\mathbf{5 0 , 5 1}}$. To make these polymeric nanoparticles appropriate for systemic circulation they should have a LCST above the human body temperature $\left(\sim 37^{\circ} \mathrm{C}\right)$. By increasing the molar ratio of the hydrophilic vinyl derivatives, a high LCST of polymeric nanoparticles within the range of $45-50^{\circ} \mathrm{C}$, can be achieved due to which such nanoparticles become stable in the body for a longer time. These polymeric nano carriers are being used for various hydrophobic drugs, anticancer/tumor agents such as Paclitaxel, Docetaxel, Rapamycin, Doxorubicin, Benzylphenylurea (BPU) compounds; phytochemicals and other natural drugs such as curcumin, curcuminoids; antiviral agents such as Aciclovir, Indinavir, Lamivudine, Stavudine etc.; antifungal agents such as Itraconazole, Ketoconazole, Miconazole, Oxiconazole etc.; antibacterial agents such as quinolones together with Ciprofloxacin, Ofloxacin, Moxifloxacin etc.; antibacterial agents such as penicillins with Cloxacillin, Benzylpenicillin, Phenylmethoxypenicillin; antitubercular agents such as rifampicin and rifapentin and anti-inflammatory agents such as Ibuprofen,

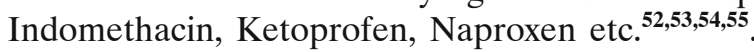

Recently nanoparticles, targeting Hepatoma cells explored the system of poly $(-\gamma$-glutamic acid $)-$ poly (lactide) block copolymers. Over the time, biodegradable nanoparticles self-possessed of amphiphilic block copolymers have attracted a significant interest as the effective drug carriers. In biodegradable and biocompatible polymer systems, $\gamma$-PGA and PLA are used to manufacture $\gamma$-PGA-PLA block copolymers through a simple combination reaction between $\gamma$-PGA and PLA to prepare self-assembled nanoparticles. Galactosamine has been found to be a conjugated targeting moiety. The $\gamma$-PGA is a naturally occurring anionic homo-polyamide that contains L-glutamic acid units joined by amide linkages between $\alpha$-amino and $\gamma$-carboxylic acid groups ${ }^{56}$. It is an exocellular polymer of some Bacillus species that is found in the fermentation broth excreted by the cell through TCA cycle.

Literature exhibits, hydrogels were constructed of two layers of poly ( $\mathrm{N}$-isopropylacrylamide) and acrylamide in the mixture of $1.75 \mathrm{M}$ poly (NIPAAm-co-AAm). The thermal behavior of this copolymeric nanoparticle NIPAAm-co-AAm hydrogels (diamond) is 0-60 min during immersion in $50^{\circ} \mathrm{C}$ and $60-90$ min during room temperature buffer. The more hydrophilic AAm formed the copolymer with NIPAAm a relatively thin surface, permitting soluble materials held within the hydrogel matrix to be more easily ejected into the surrounding solution during the burst of the particles. The LCST of the NIPAAm-co-AAm copolymer hydrogels can hang from $32-65^{\circ} \mathrm{C}$, based on the amount of AAm holding in the copolymer. A copolymer hydrogel having a LCST of approximately $40^{\circ} \mathrm{C}$ including of $95 \%$ NIPAAM and $5 \%$ AAM ${ }^{57,58,59}$.

Recently, a poly (ester amide) (PEA) stimuli responsive copolymer has been formed. In PEA polymer has a stimuli responsive backbone of amino acids which has no limitation to anti-proliferative agents and anti-inflammatory agents. The polymer PEA includes an amino acid, a diol, and a diacid. There are two forms of this PEA polymer binding with lysine substituent, one is PEA-Benzyl and another PEA-Tempo. The 90-day in vivo study of the degradation of a PEA-Benzyl was evaluated by the help of the anti-proliferative agent Everolimus. The dose of the Everolimus was $190 \mu \mathrm{g} / \mathrm{cm}^{2}$. The percentage mass loss of PEA-Benzyl between 0 and 90 days shows the degradation rate when there are low and high levels of inflammation $^{\mathbf{6 0 , 6 1}}$.

\section{pH-SENSITIVE POLYMERIC NANOPARTICLES}

For research progress in the area of the regulated drug delivery system, scientists are developing various novel approaches to deliver the drugs at a specific site of action into the body. The $\mathrm{pH}$-sensitive polymeric nanoparticles based drug delivery method is the most promising technique among them. The $\mathrm{pH}$-responsive polymeric nanoparticles could release the drug in a slightly acidic environment, which occurs in inflammatory tissues, solid tumors and intracellular endosomal compartments. There are the possibilities of the cytoplasmic drug delivery of internalized macromolecules (e.g. oligonucleic acid, DNA, proteins and polymer-antibody complex) [Fig. 3]. For intracellular carriers of the drug a polymeric system of synthetic polymers that is polyanions (acrylic acid derivatives) and polycations (poly ethyleneamine and chitosan mixture) has been developed ${ }^{62}$.

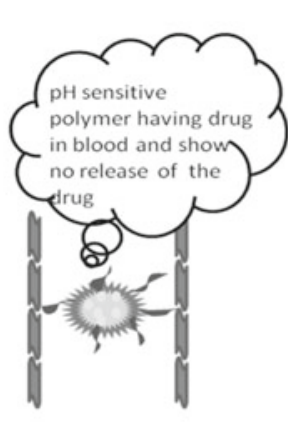

Blood pH 7.4

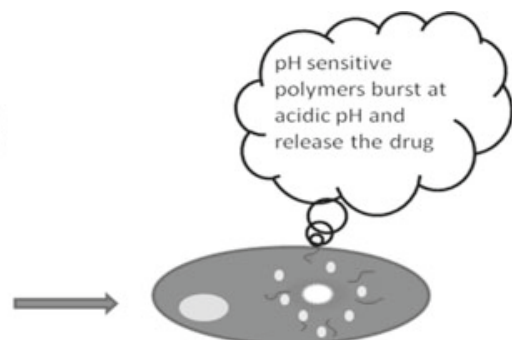

Tumor Intracellular pH 5.0-6.0
Figure 3. Delivery of the drug through $\mathrm{pH}$ sensitive nanoparticles

In a recent publication, the $\mathrm{pH}$-sensitive polymeric micelles developed using poly (L-histidine)-poly (ethylene glycol) block copolymer and poly (L-lactic acid)-poly (ethylene glycol) block copolymer ${ }^{63}$ that release the drug in an acidic environment, but not in the blood. Though, the solid tumors have acidic environment, these $\mathrm{pH}$ sensitive polymeric micelles are beneficial to drug release at appropriate site for curing cancer, including those cancers which show the multidrug resistance. Many types of anticancer drugs were used by the inventors through this micellar system e.g. adramycine, doxorubicine etc.

The extracellular $\mathrm{pH}\left(\mathrm{pH}_{\mathrm{e}}\right)$ of the tumor is a consistently distinguishing phenotype of maximum solid tumors to surrounding normal tissues. The measured $\mathrm{pH}$ values of most solid tumors in patients are found to be in the range of $\mathrm{pH} 5.7$ to $\mathrm{pH} 7.8$ with an average value of 7.0. According to this study, $\mathrm{pH}$ - sensitive polymeric micelles contain mixture of poly (L-histidine)-poly (ethylene glycol) block copolymer and an amphiphilic block copolymer $^{\mathbf{6 4 , 6 5}}$. These types of polymeric micelles are prolonged in the blood, but release the drug carried in such 
micelles upon reaching the acidic state, e.g. environment of the solid tumors for curing cancer. The $\mathrm{pH}$ of this polymeric micelle was measured by light transmittance of the micellar solution, with a concentration of $0.1 \mathrm{~g} / \mathrm{L}$, at $\mathrm{pH} 8.0$ in a buffer solution of $\mathrm{NaOH}-\mathrm{Na}_{2} \mathrm{~B}_{4} \mathrm{O}_{7}$ (ionic strength -0.1 ) gradually reduced by mixing $0.01 \mathrm{~N} \mathrm{HCl}$ solution.

Phagosome, endosome, or lysosome have an acidic $\mathrm{pH}$, therefore, a novel regulated drug delivery system that permits the drug release of an agent to be delivered in response to acidic $\mathrm{pH}$, is required. A new regulated drug delivery system includes poly (beta-amino esters) to facilitate the disruption of nanoparticles and for releasing their drugs. Some synthetic polymers are mixed such as (polyesters, polyanhydrides, polyamides, polyureas, polyethers, polyacrylates, polymethacrylates, poly (beta-amino esters), polycarbonates, polycarbamates,) and copolymers to build the carrier triggerable. For making a $\mathrm{pH}$ triggerable particle, there is an essentiality of a $\mathrm{pH}$ triggering agent that is a chemical compound with a pka below pH 7.0 e.g. poly (beta-aminoester) ${ }^{\mathbf{6 6 , 6 7}}$.

The $\mathrm{pH}$ sensitive polymers contain adornment acidic or basic groups to accept or donate protons in response to environmental $\mathrm{pH}$. Hydrogel puffiness raise as the external pH swells up in the case of weak acidic groups, but shrink if polymer contains weak basic groups ${ }^{\mathbf{6 8 , 6 9}}$. A large number of anionic $\mathrm{pH}$-sensitive polymeric nanoparticles contain polyacrylic acid (PAA) e.g. $\left(\right.$ Carbopol $\left.^{\circledR}\right)$. A few more polymers such as polymethacrylic acid (PMAA), poly (ethylene amine), poly (L-lysine), and poly ( $N, N$ dimethyl aminoethyl methacrylamide) are also added in this series for their use in a regulated drug delivery ${ }^{\mathbf{7 0}}$. The monomers of acrylic acid and methacrylic acid are the $\mathrm{pH}$ sensitive poly acids having carboxylic acidcontaining polymers. A copolymer hydrogel which made 90\% methyl methacrylate (MMA) and $10 \%$ acrylic acid (AA) for the controlled drug delivery, is studied. The polymer matrix swells when the environment reaches $\mathrm{pH} 8.5$, releasing the active drug. Alginate gel beads or particles are diverse, sustain release $\mathrm{pH}$ sensitive polymeric nanoparticles. The formulation of alginate gel beads release the drug sustainable by co-precipitation. There is good protein stability and loading of the drug is high. In this invention the permeability percentage increase of theophylline is reduced with increasing the ionic strength as temperature increases from $20^{\circ} \mathrm{C}$ to $45^{\circ} \mathrm{C}$. The permeability increment with the presence of monovalent ions, $\mathrm{K}^{+}$is always lower in the presence of divalent ions, $\mathrm{Ca}^{2+}$. This surveillance recommends that the infiltration is responsive to the divalent ions.

In an advanced research, a pH-sensitive (meth) acrylic copolymer is developed having the molecular weight between 1000-50000 g/mol using 20-65\% methacrylic acid units and 80-35\% C1-C18 - alkyl ester of (meth) acrylic acid accordingly by weight ${ }^{71,72}$. In a cytotoxicity test with human red blood cells, the formula explore the $60 \%$ haemolysis at $\mathrm{pH} 5.5$ and less than $5 \%$ haemolysis

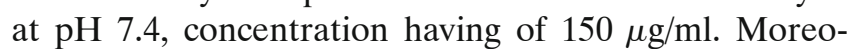
ver, the process of this formula has failed in response to the upper limit of the molecular weight. As poly (meth) acrylates polymers are within the range of a given molecular weight they are easily filtrated through the kidney. The kidney permits secretion of molecules that have adopted the upper limit of $50000 \mathrm{~g} / \mathrm{mole}$. Hence the low molecular weight polymers could show much better hemolytic activities than the high molecular weight molecules.

In a unique field of science, new conductive dispersions are fashioned of polypyrrole and the colloid-forming polymeric acid by the accumulation of highly conductive additives. For the reason that dispersions with a comparatively high $\mathrm{pH}$ can be formed, the conductive additives, are not assaulted by the acid in the dispersion like metal additives. However, electrically conducting polypyrrole is fashioned on the colloidal exterior due to the colloidal nature of the polymeric acid, containing the exterior predominately including acid groups ${ }^{73,74}$. Due to this inimitable configuration, only a low weight percentage of highly conductive additives are needed to achieve the percolation doorstep. Examples of appropriate conductive additives include metal particles and nanoparticles, carbon nanotubes, carbon nanoparticles, graphite particles, and their combinations.

There are diblock or triblock copolymers of PLA or PLGA and PEG, whose functional groups are ionize able on one or both ends of the hydrogel polymeric chains. The mixture of hydroxyl terminated and carboxylic $30 \%(\mathrm{w} / \mathrm{w})$ solution completed PLGA-PEG-PLGA exhibit sustained release of Fc-leptin over one week time period. The recently grafted and block co-polymeric nanoparticles which hold both type of polymeric nanoparticles (a temperature sensitive and $\mathrm{pH}$ sensitive) were described ${ }^{\mathbf{7 5 , 7 6}}$. A synthetic carboxylic acid-containing $\mathrm{pH}$-sensitive polymer which is made from polymerizable carboxylic acids, with acrylic acid, methacrylic acid and ethacrylic acid. Polymeric systems grasping PVA-g-PAAc grafted copolymers display appreciably greater lysozyme release at pH 7.4 (PBS buffer) than at pH 5.0 (2- $[\mathrm{N}$ morpholino]-ethanesulfonic acid (MES buffer). Gel plays an important role in drug release, as the swelling of the drug from the gel is better, the diffusion of the drug is better.

Many smart polymeric nanoparticles are recognized to respond to a mixture of two or more stimuli. This provides an obvious advantage since it introduces more means of release profile control. Recently, for the release of sustained drug delivery of biologically active agents, $\mathrm{pH}$ sensitive/thermosensitive biodegradable hydrogels were described $^{77,78}$. In a new research, such type of controlled drug delivery system has been developed to overcome the serious endocrine disorder diabetic mellitus. The $\mathrm{pH}$ sensitive particle that is formed is in the range of 30-100nm and includes the various polymers, fatty acid, protein, surfactant and cross linking agent. This is the oral formulation of the insulin and saves insulin from the acidic $\mathrm{pH}$ environment and digestive enzymes due to the shrinkage at gastric $\mathrm{pH}$ and the stability of encapsulated insulin is found 5-7 month at the temperature $4^{\circ} \mathrm{C}^{\mathbf{7 9}, 80}$.

In a recent study, chitosan (CS) that is a cationic polysaccharide, non-toxic and soft - tissue compatible, has a unique feature of attaching to the mucosal surface and opens the tight junctions between epithelial cells. Chitosans should be dissolved in an acetic acid solution at $\mathrm{pH}$ value of 4.0 and lower than this due to large molecular weight. Preserving the bioactivity of the peptides or proteins is dependent upon the encapsulation 
at the physiological $\mathrm{pH}$ ranges and the low molecular weight and the polycationic features are used to make the better solubility at a $\mathrm{pH}$ value. Therefore, the low molecular weight chitosan-y-PGA (poly glutamic acid) nanoparticles have been prepared using cellulose $\mathrm{e}^{51,81,82}$.

\section{CONCLUSION}

For the last many years, numerous strategies have been developed using polymeric nanoparticles as a drug delivery system for the sustained drug release. A lot of stimuli responsive polymeric systems are being used in clinical treatment having their commercial production and many are in channel. However, for the development, there are a number of polymeric regulated drug delivery systems which are still far from principle. For instance, these polymeric systems are prompt to the altering metabolic condition in patients. Therefore, a number of thoughts take account of biocompatibilities, break open release, a stimuli responsive time, most favorable release rate imitation, prolong circulation time of drug, and formulation issues and challenges. Our review on numerous advanced studies explores the role of stimuli responsive polymeric nanoparticles for the regulated drug delivery. These types of polymeric nanoparticles systems have the prospective approach for the regulated release of various drugs. Temperature and $\mathrm{pH}$ sensitive polymeric systems have advantages over the disease by delivering a drug into the site in a specific sustained release manner which is a part of a current development in a novel drug delivery system. Though, tremendous opportunities are left to find out the appropriate stimuli responsive drug delivery systems, which are biodegradable, biocompatible, site specific sustained release, due to these characteristics it makes easier to control the delivery and stability of the drug into body.

\section{LITERATURE CITED}

1. Johnson, B.F.G. (2003). Topics in Catalysis, Chem. \& Mater. Sci. 24(1-4), 147-159. DOI: 10.1023/B:TOCA.0000003086.83434.b6.

2. Khanna, V.K. (2008). Nanoparticle-based Sensors, Defence Sci. J. 58(5), 608-616.

3. Hoshino, A., Fujioka, K. \& Oku, T. (2004). Physicochemical properties and cellular toxicity of nanocrystal quantum dots depend on their surface modification. Nano Lett. 4(11), 2163-2169. DOI: 10.1021/nl048715d.

4. Torchilin, V. P. (2001). Structure and design of polymeric surfactant-based drug delivery systems, J Cont. Rel. 73(2-3), 137-172. DOI:10.1016/S0168-3659(01)00299-1.

5. Connor, E. E., Mwamuka, J., Gole, A., Murphy, C.J. \& Wyatt, M.D. (2005). Gold nanoparticles are taken up by human cells but do not cause acute cytotoxicity. Small. 1(3), 325-327. DOI: $10.1002 / \mathrm{smll} .200400093$.

6. Wu, X.Y. \& Lee, P.I. (1993). Preparation and characterization of thermal and $\mathrm{pH}$ sensitive nanospheres. Pharm. Res. 10(10), 1544-1547. DOI: 10.1023/A:1018900114881.

7. Huang, M., Khor, E. \& Lim, L.Y. (2004). Uptake and cytotoxicity of chitosan molecules and nanoparticles: effects of molecular weight and degree of deacetylation. Pharm Res. 21:344-353. DOI: 10.1023/B:PHAM.0000016249.52831.a5.

8. Kabanov, A. V. \& Alakhov, V. Y. (2002). Pluronic block copolymers in drug delivery: From micellar nanocontainers to biological response modifiers. Crit. Rev. Ther. Drug Carrier Syst. 19(1), 1-72.
9. Chavanpatil, M.D., Patil, Y. \& Panyam, J. (2006). Susceptibility of nanoparticle-encapsulated paclitaxel to p-glycoprotein-mediated drug efflux. Int. J. Pharm. 320, 150-156. DOI:10.1016/j.ijpharm.2006.03.045.

10. Pettit, D.K. \& Gombotz, W.R. (1998). The development of site-specific drug-delivery systems for protein and peptide biopharmaceuticals, Trends in Biotech. 16(8), 343-349. DOI:10.1016/S0167-7799(98)01186-X.

11. Bellocq, N.C., Pun, S.H., Jensen, G.S. \& Davis, M.E. (2003). Transferrin-containing, cyclodextrin polymer-based particles for tumor-targeted gene delivery. Bioconjug. Chem. 14(6), 1122-1132. DOI: 10.1021/bc034125f.

12. Feng, S.S. (2004). Nanoparticles of biodegradable polymers for new-concept chemotherapy, Expert Rev. Med. Dev. 1(1), 115-125. DOI:10.1586/17434440.1.1.115.

13. Qiao, W., Wang, B., Wang, Y., Yang, L., Zhang, Y. \& Shao, P. (2010). Cancer Therapy Based on Nanomaterials and Nanocarrier Systems, J. Nanomater. Article ID 796303, 9. DOI:10.1155/2010/796303.

14. Sona, P.S. (2010). Nanoparticulate Drug Delivery Systems for the Treatment of Diabetes, Digest J. Nanomater. \& Biostruc. 5(2), 411-418.

15. Vlerken, L.E.V. \& Amiji, M.M. (2006). Multi-functional polymeric nanoparticles for tumor-targeted drug delivery. Expert Opi. Drug Deliv. 3(2), 205-16. DOI:10.1517/17425247.3.2.205.

16. Avgoustakis, K. (2004). Pegylated poly (lactide) and poly (lactide-co-glycolide) nanoparticles: preparation, properties and possible application in drug delivery, Curr. Drug Deliv. 1(4), 321-333. DOI: 10.2174/1567201043334605.

17. Yam, F., Wu, X. Y. \& Zhang, Q. (2000). A novel composite membrane for temperature and $\mathrm{pH}$ responsive permeation, in: Controlled Drug Delivery: Designing Technology for the Future (263-272). Ed K. Park, ACS, Washington, DC.

18. Senior, J. \& Gregoriadis, G. (1982). Is half-life of circulating small unilamellar liposomes determined by changes in their permeability? FEBS Lett. 145(1), 109-114. DOI. org/10.1016/0014-5793(82)81216-7.

19. Shenoy, D., Fu, W., Li, J., Crasto, C., Jones, G., Dimarzio, C., Sridhar, S. \& Amiji, M. (2006). Surface functionalization of gold nanoparticles using hetero-bifunctional poly (ethylene glycol) spacer for intracellular tracking and delivery, Int $J \mathrm{Na}$ nomed. 1(1), 51-57. DOI: 10.2147/nano.2006.1.1.51.

20. Edward, T. \& Yeop, S.J. (2002). U.S. Patent No. 20,070,190,160. Washington, D.C.: U.S. Patent and Trademark Office.

21. Yin, H., Lee, E. S., Kim, D., Lee, K.H., Oh, K.T. \& Bae, Y.H. (2008). Physicochemical characteristics of pH-sensitive poly (L-Histidine)-b-poly (ethylene glycol) / poly (L-lactic acid)-b-poly (ethylene glycol) mixed micelles, J. Control. Rel. 126(2), 130-138. DOI:10.1016/j.jconrel.2007.11.014.

22. Yoshida, R., Kaneko, Y., Sakai, K., Okano, T., Sakurai, Y., Bae, Y.H. \& Kim, S.W. (1994). Positive thermosensitive pulsatile drug release using negative thermosensitive hydrogels, $J$. Contro. Rel. 32(1), 97-102. DOI:10.1016/0168-3659(94)90229-1.

23. Seymour, L.W., Duncan, R., Strohalm, J. \& Kopecek, J. (1987). Effect of molecular weight (MW) of N-(2-hydroxypropyl) methacrylamide copolymers on body distribution and rate of excretion after subcutaneous, intraperitoneal, and intravenous administration to rats, J. Biomed. Mater. Res. 21(11), 1341-1358. DOI: $10.1002 / \mathrm{jbm} .820211106$.

24. Shefer, S. \& Shefer, A. (2004). U.S. Patent No. 20,040,062,778. Washington, D.C.: U.S. Patent and Trademark Office.

25. Yang, T.H. (2008). Recent Applications of Polyacrylamide as Biomaterials, Rec. Pat. Mater. Sci. 1(1), 29-40. DOI: $10.2174 / 1874465610801010029$.

26. Yong, L.I.Y., Qing, D.H., Kang, W., DongLu, S.H.I., Zheng, Z. X. \& Xi, Z. R. (2010). Stimulus-responsive polymeric Nanoparticles for biomedical applications, Sci. China Chem. 53(3), 447-457. DOI: 10.1007/s11426-010-0101-4. 
27. Song, M., Guo, D., Pan, C., Jiang, H., Chen, C., Zhang, R., Gu, Z. \& Wang, X. (2008). The application of poly(N-isopropylacrylamide)-co-polystyrene nanofibers as an additive agent to facilitate the cellular uptake of an anticancer drug, Nanotechno. 19(16), 165102. DOI: 10.1088/0957-4484/19/16/165102.

28. Bromberg, L., Temchenko, M. \& Hatton, T.A. (2002). Dually Responsive Microgels from Polyether-Modified Poly (acrylic acid): Swelling and Drug Loading, Langmuir. 18(12), 4944-4952. DOI: 10.1021/la0118681.

29. Izumi, S. \& Kunihiko, T. (1985). U. S. Patent No. 4,536,387. Washington, D.C.: U.S. Patent and Trademark Office.

30. Youwei, Z. \& Ming, J. (2006). New approaches to stimuli-responsive polymeric micelles and hollow spheres, Front. Chem. China. 1(5), 364-368. DOI: 10.1007/s11458-006-0049-2.

31. Grodzinski, J.J. (1999). Biomedical application of functional polymers, React. \& Funct. Poly. 39, 99-138.

32. Sung, Y.K. \& Kim, S.W. (2000). Advances in biodegradable polymers for drug delivery systems, Korean Poly. J. 8(5), 199-208.

33. Knoop, R.J.I., Geus, M.D., Habraken, G.J.M., Koning, C.M., Menzel, H. \& Heise, A. (2010). Stimuli Responsive Peptide Conjugated Polymer Nanoparticles, Macromol. 43(9), 4126-4132. DOI: 10.1021/ma100327p.

34. Wei, H., Zhang, X. Z., Zhou, Y., Cheng, S. X. \& Zhuo, R. X. (2006). Self-assembled thermoresponsive micelles of poly(N-isopropylacrylamide-b-methyl methacrylate), Biomater. 27(9), 2028-2034. DOI:10.1016/j.biomaterials.2005.09.028.

35. Yu, W.X. \& Frank, Y. (2003). U. S. Patent No. 6565,872. Washington, D.C.: U.S. Patent and Trademark Office.

36. Wang, J., Cheng, Y. \& Xu, T. (2008). Current Patents of Dendrimers and Hyperbranched Polymers in Membranes, Recent Pat. Chem. Enginee. 1(1), 41-51.

37. Erathodiyil, N., Reddy, G.R. \& Ham, Y. (2007). U. S. Patent No. 20,070,009,441. Washington, D.C.: U.S. Patent and Trademark Office.

38. Lyer, A.K., Khaled, G., Fang, J \& Maeda, H. (2006). Exploiting the enhanced permeability and retention effect for tumor targeting, Drug Discov. Today. 11(17-18), 812-818. DOI:10.1016/j.drudis.2006.07.005.

39. Chang, J.S., Chang, K.L.B., Hwang, D.F. \& Kong, Z. L. (2007). In vitro cytotoxicity of silica nanoparticles at high concentrations strongly depends on the metabolic activity type of the cell line, Environ. Sci. Technol. 41(6), 2064-2068. DOI: 10.1021/es062347t

40. Linhardt, J.G., Raiche, A.T. \& Salmone, C. (2009). U. S. Patent No. 20,090,117,189. Washington, D.C.: U.S. Patent and Trademark Office.

41. Zhang, G., Desnoyer J.R., Stewart, G., Kezis, M. \& Hossainy, S.F.A. (2008). U.S. Patent No. 20,080,057,024. Washington, D.C.: U.S. Patent and Trademark Office.

42. West, J.L., Sershen, S,R. \& Halas, N.J. (2002). U. S. Patent No. 6,428,811. Washington, D.C.: U.S. Patent and Trademark Office.

43. Rapoport, N. (2007). Physical stimuli-responsive polymeric micelles for anti-cancer drug delivery, Prog. Poly. Sci. 32(8-9), 962-990. DOI:10.1016/j.progpolymsci.2007.05.009.

44. Hatefi, A. \& Amsden, B. (2002). Biodegradable inject able in situ forming drug delivery systems, J Control. Rel. 80(1-3), 9-28. DOI:10.1016/S0168-3659(02)00008-1.

45. Yin, H., Lee, E.S., Kim, D., Lee, K.H., Oh, K.T. \& Bae, Y.H. (2008). Physicochemical characteristics of $\mathrm{pH}$-sensitive poly (l-Histidine)-b-poly (ethylene glycol)/poly (l-Lactide)-b-poly (ethylene glycol) mixed micelles, J. Control. Rel. 126(2), 130-138. DOI:10.1016/j.jconrel.2007.11.014.

46. Ichikawa, H. \& Fukumori, Y. (2000). A novel positively thermosensitive controlled release microcapsule with membrane of nano-sized poly (N-isopropylacrylamie) gel ispesed in ethylcellulose matrix, J. Control. Rel. 63(1-2), 107-119.

47. Donald, O., Peppas, E. \& Nicholas, A. (2008). U.S. Patent No. 20,080,138,430. Washington, D.C.: U.S. Patent and
Trademark Office.

48. Park, Y.S., Ito, Y. \& Imanishi, Y. (1998). Permeation control through porous membranes immobilized with thermosensitive polymer, Langmuir. 14(4), 910-914. DOI: 10.1021/ la970866r.

49. Mitra, S., Gaur, U., Ghosh, P.C. \& Maitra, A.N. (2001). Tumour Targeted Delivery of Encapsulated Dextran-Doxorubicin Conjugate Using Chitosan Nanoparticles as Carrier. J. Control. Rel. 74(1-6), 317-323. DOI: 10.1016/S0168-3659(01)00342-X.

50. Chen, H.C. \& Chatterjee, Y. (2006). U. S. Patent No. 7,081,489. Washington, D.C.: U.S. Patent and Trademark Office.

51. Singer, J. W., Baker, B., De Vries, P., Kumar, A., Shaffer, S., Vawter, E., Bolton, M. \& Garzone, P. (2003). Poly-(L)-glutamic acid-paclitaxel (CT-2103) [XYOTAX (TM)], a biodegradable polymeric drug conjugate-Characterization, preclinical pharmacology, and preliminary clinical data, $A d v$. Exp. Med. Biol. 519, 81-99. DOI: 10.1007/0-306-47932-X 6.

52. Zhao, M., Zabelina, Y., Rudek, M.A., Wolff, A.C. \& Baker, S.D. (2003). A rapid and sensitive method for determination of dimethyl benzoylphenyl urea in human plasma by using LC/MS/MS, J. Pharmaceu. \& Biomed. Anal. 33(4), 725-733. DOI:10.1016/S0731-7085(03)00424-2.

53. Sung \& Hsing-Wen. (2006). U. S. Patent No. 20,060,115,537. Washington, D.C.: U.S. Patent and Trademark Office.

54. Na, K., Lee, K.H., Lee, D.H. \& Bae, Y.H. (2006). Biodegradable thermo-sensitive nanoparticles from poly(L-lactic acid)/poly(ethylene glycol) alternating multi-block copolymer for potential anti-cancer drug carrier, Eur. J. Pharm. Sci. 27(2-3), 115-122. DOI:10.1016/j.ejps.2005.08.012.

55. Stayton, P.S., Hoffman, S.S. \& Xiangchun, Y. (2001). U.S. Patent No. 20,070,224,241. Washington, D.C.: U.S. Patent and Trademark Office.

56. Chen, D., Jiang, M. \& Huisheng, P. (2007). U. S. Patent No. 7,166,306. Washington, D.C.: U.S. Patent and Trademark Office.

57. Des Rieux, A., Fievez, V., Garinot, M., Schneider, Y.J. \& Preat, V. (2006). Nanoparticles as potential oral delivery systems of proteins and vaccines: a mechanistic approach, $J$. Control. Rel. 116(1), 1-27. DOI:10.1016/j.jconrel.2006.08.013.

58. Ko, J., Park, K., Kim, Y.S., Kim, M.S., Han, J.K., Kim, K., Park, R.W., Kim, I.S., Song, H.K. \& Lee, D.S. (2007). Kwon IC. Tumoral acidic extracellular $\mathrm{pH}$ targeting of $\mathrm{pH}$-responsive MPEG-poly (beta-amino ester) block copolymer micelles for cancer therapy, J. Control. Rel. 123(2), 109-115. DOI:10.1016/j. jconrel.2007.07.012.

59. Wright, D.C. (1998). U.S. Patent No. 5,795,582. Washington, D.C.: U.S. Patent and Trademark Office.

60. Dunn, R.L., Garrett, J.S. \& Ravivarapu, H. (2004). U.S. Patent No. 6,773,714. Washington, D.C.: U.S. Patent and Trademark Office.

61. Harada, M., Sakakibara, H., Yano, T., Suzuki, T. \& Okuno, S. (2000). Determinants for the Drug Release from T-0128, Camptothecin Analogue-Carboxymethyl Dextran Conjugate, J. Control. Rel. 69(3), 399-412. DOI:10.1016/S01683659(00)00321-7.

62. Chen, D. Jiang, M. Peng. \& Huisheng. (2007). U.S. Patent No. 7,166,306. Washington, D.C.: U.S. Patent and Trademark Office.

63. Ulbrich, K. \& Subr, V. (2004). Polymeric anticancer drugs with pH-controlled activation, Adv. Drug Deliv. Rev. 56(7), 1023-1050. DOI:10.1016/j.addr.2003.10.040.

64. Kramer, M., Stumbe, J.F., Turk, H., Krause, S., Komp, A., Delineau, L., Prokhorova, S., Kautz, H. \& Haag, R. (2002). $\mathrm{pH}$-responsive molecular nanocarriers based on dendritic core-shell architectures, Angew. Chem. Int. Ed. 41(22), 4252-4256. DOI: 10.1002/1521-3773(20021115.

65. Lee, E.S., Na, K. \& Bae, Y.H. (2005). Super pH-sensitive multifunctional polymeric micelle, Nano Lett. 5(2), 325-329. DOI: $10.1021 / \mathrm{nl} 0479987$.

66. Little, S.R., Lynn, D.M. \& Anderson, D.G. (2005). U.S. 
Patent No. 20,050,245,049. Washington, D.C.: U.S. Patent and Trademark Office.

67. Kim, J.J. \& Park, K. (2001). Modulated insulin delivery from glucose-sensitive hydrogel dosage forms, J. Control. Rel. 77(1-2), 39-47. DOI:10.1016/S0168-3659(01)00447-3.

68. Lavasanifar, A., Samuel, J. \& Kwon, G.S. (2002). Poly (ethylene oxide)-block-poly (L-amino acid) micelles for drug delivery, Adv. Drug Deliv. Rev. 54(2), 169-190. DOI:10.1016/ S0169-409X(02)00015-7.

69. Lee, I. \& Srivastava, D. (2008). U.S. Patent No. 20,080,176,074. Washington, D.C.: U.S. Patent and Trademark Office.

70. Chen, Mei-chin, T. \& Hos1heng. (2008). U.S. Patent No. 20,080,160,078. Washington, D.C.: U.S. Patent and Trademark Office.

71. Hubbell, J.H., Pathak, C. P. and Sawhney, A. S. (1999). U. S. Patent No. 5,986,043. Washington, D.C.: U.S. Patent and Trademark Office.

72. Petereit, Hans-ulrich, \& Meier. (2005). U.S. Patent No. 20,050,154,165. Washington, D.C.: U.S. Patent and Trademark Office.

73. Monahan, S.D., Wolff, J.A. and Hagstrom, J.E. (2007). U.S. Patent No. 7,208,314. Washington, D.C.: U.S. Patent and Trademark Office.

74. Ohya, Y., Oue, H., Nagatomi, K. \& Ouchi, T. (2001). Design of Macromolecular Prodrug of Cisplatin Using Dextran with Branched Galactose Units as Targeting Moieties to Hepatoma Cells, Biomacro, 2(3), 927-933. DOI: http://dx.doi. org/10.1021/bm010053o.

75. Pendri, A., Conover, C.D. \& Greenwald, R.B. (1998). Antitumor activity of paclitaxel-2-glycinate conjugated to poly(ethylene glycol): a water-soluble prodrug, Anticancer Drug Des. 13(5), 387-395.

76. Rathi, R.C., Zentner, G.M. and Jeong, B. (2000). U.S. Patent No. 20,006,117,949. Washington, D.C.: U.S. Patent and Trademark Office.

77. Heffernan, M.J. \& Murthy, N. (2005). Polyketal nanoparticles: A new $\mathrm{pH}$-sensitive biodegradable drug delivery vehicle, Bioconjug. Chem. 16(6), 1340-1342. DOI: 10.1021/bc050176w.

78. Sharma, P.C. (2009). U. S. Patent No. 20,090,098,205. Washington, D.C.: U.S. Patent and Trademark Office.

79. Tomlinson, R., Klee, M., Garrett, S., Heller, S., Duncan, R. \& Brocchini, S. (2002). Pendent chain functionalized polyacetals that display $\mathrm{pH}$-dependent degradation: A platform for the development of novel polymer therapeutics, Macromole. 35(2), 473-480. DOI: 10.1021/ma0108867.

80. Chen, C.J., Haik, Y. \& Chatterjee, J. (2006). U. S. Patent No. 7,081,489. Washington, D.C.: U.S. Patent and Trademark Office.

81. Wenzel, J.G.W., Balaji, K.S.S., Koushik, K., Navarre, C., Duran, S.H., Rahe, C.H. \& Kompella, U.B. (2002). Pluronic F127 gelformulations of deslorelin and GnRH reduce drug degradation and sustain drug release and effect in cattle. $J$. Control. Rel. 85, 51-59. DOI:10.1016/S0168-3659(02)00271-7.

82. Bae, Y.H., Na, K. \& Lee (2005). U.S. Patent No. 20,050,186,263. Washington, D.C.: U.S. Patent and Trademark Office. 